\title{
Pseudo-UP Ideals and Pseudo-UP Filters in Pseudo-UP Algebras
}

\author{
Daniel A. Romano*
}

\begin{abstract} of pseudo-UP ideals and pseudo-UP filters in pseudo-UP algebras.

Keywords: UP-algebra, Pseudo-UP algebra, Pseudo-UP ideal, Pseudo-UP filter.

AMS Subject Classification (2020): 03G25.

${ }^{*}$ Corresponding author
\end{abstract}

The notion of pseudo-UP algebras is introduced and analyzed in our forthcoming article as a generalization of UP-algebras. In this article, as a continuation of the foregoing, we introduce and analyze concepts

\section{Introduction}

The concept of pseudo-BCK algebras was introduced in [3] by G. Georgescu and A. Iorgulescu as an extension of BCK algebra. The pseudo-ideals of pseudo-BCK algebras were introduced in 2003 by Y. B. Jun, M. Kondo and K. H. Kim in article [8]. The notion of pseudo-BCI algebras was introduced and analyzed in [1] by W. A. Dudek and Y. $\mathrm{B}$. Jun as a generalization of BCI-algebras. The notion of pseudo-BCI ideals in pseudo-BCI algebras is introduced 2006 in [9] by Y. B. Jun, H. S. Kim and J. Neggers. These substructures in pseudo-BCK and pseudo-BCI algebras have been studied by several authors such as, for example, K. J. Lee and C. H. Park. [11] and G. Dymek [2]. These algebraic structures has been in the focus of many authors (for example, see $[4,6,7,19]$ ).

Iampan [5] introduced a new algebraic structure which is called UP-algebras as a generalization of KU-algebras. Somjanta et al. [18] introduced the notion of UP-filters of UP-algebras. The concept of proper UP-filters in such algebras this author introduced 2018 in [12]. In addition, some new types of UP-filters in UP-algebras were introduced by Y. B. Jun and A. Iampan in [10].

In our forthcoming article [17], we introduced the concept of pseudo-UP algebras and some properties of pseudo-UP algebras are studied. In this article, as a continuation of the foregoing, we introduce and analyze the concepts of pseudo-UP ideals and pseudo-UP filters in pseudo-UP algebras.

\section{Preliminaries}

In this section we will describe some elements of UP-algebras from the literature [5] necessary for our intentions in this text.

Definition 2.1. ([5]) An algebra $A=(A, \cdot, 0)$ of type $(2,0)$ is called a UP-algebra where $A$ is a nonempty set, ${ }^{\prime} \cdot{ }^{\prime}$ is a binary operation on $A$, and 0 is a fixed element of $A$ (i.e. a nullary operation) if it satisfies the following axioms:

$(\mathrm{UP}-1)(\forall x, y \in A)((y \cdot z) \cdot((x \cdot y) \cdot(x \cdot z))=0)$,

(UP-2) $(\forall x \in A)(0 \cdot x=x)$,

(UP-3) $(\forall x \in A)(x \cdot 0=0)$, and

(UP-4) $(\forall x, y \in A)((x \cdot y=0 \wedge y \cdot x=0) \Longrightarrow x=y)$.

Received : 07-10-2019, Accepted : 20-02-2020 
Definition 2.2. ([17]) A pseudo-UP algebra is a structure $\mathfrak{A}=((A, \leqslant), \cdot, *, 0)$, where ${ }^{\prime} \leqslant{ }^{\prime}$ is a binary relation on a set $A,{ }^{\prime} \cdot{ }^{\prime}$ and ' $*$ ' are internal binary operations on $A$ and ${ }^{\prime} 0$ ' is an element of $A$, verifying the following axioms:

$(\mathrm{pUP}-1)(\forall x, y z \in A)(y \cdot z \leqslant(x \cdot y) *(x \cdot z) \wedge y * z \leqslant(x * y) \cdot(x * z))$;

(pUP-4) $(\forall x, y \in A)((x \leqslant y \wedge y \leqslant x) \Longrightarrow x=y)$;

(pUP-5) $(\forall x, y \in A)((y \cdot 0) * x=x \wedge(y * 0) \cdot x=x)$ and

(pUP-6) $(\forall x, y \in A)((x \leqslant y \Longleftrightarrow x \cdot y=0) \wedge(x \leqslant y \Longleftrightarrow x * y=0))$.

\section{Pseudo-UP ideals and pseudo-UP filters in a pseudo-UP algebra}

In the following definition, we introduce the concept of pseudo-UP ideals in pseudo-UP algebras

Definition 3.1. A nonempty subset $J$ of a pseudo-UP algebra $\mathfrak{A}$ is called a pseudo-UP ideal of $\mathfrak{A}$ if it satisfies

(pJ1) $0 \in J$

$(\mathrm{pJ} 2)(\forall x, y, z \in A)((x \cdot(y * z) \in J \wedge y \in J) \Longrightarrow x \cdot z \in J)$ and

$(\mathrm{pJ} 3)(\forall x, y, z \in A)((x *(y \cdot z) \in J \wedge y \in J) \Longrightarrow x * z \in J)$.

The following theorem describes the characteristic features of these substructures

Theorem 3.1. Let $J$ be a pseudo-UP ideal in a pseudo-UP algebra $\mathfrak{A}$. Then:

(1) $(\forall y, z \in A)((y * z \in J \wedge y \in J) \Longrightarrow z \in J)$;

(2) $(\forall x, y \in A)(y \in J \Longrightarrow x * y \in J)$;

(3) $(\forall y, z \in A)((y \cdot z \in J \wedge y \in J) \Longrightarrow z \in J)$ and

(4) $(\forall x, y \in A)(y \in J \Longrightarrow x \cdot y \in J)$.

Proof. Since Equations (3) and (4) can be proved in a similar way to the proofs of equality (1) and (2), we will only show the last two mentioned.

Putting $x=0$ in (pJ2), we obtain (1) with respect to equality (9) in the article [17].

Putting $z=y$ in (pJ3), we obtain (2) with respect to (pJ1) and to equalities (10) and (8) in the article [17].

Corollary 3.1. Let $J$ be a pseudo-UP ideal in a pseudo-UP algebra $\mathfrak{A}$. Then:

(5) $(\forall y, z \in A)((y \leqslant z \wedge y \in J) \Longrightarrow z \in J)$.

Proof. Let $y, z \in A$ be arbitrary elements such that $y \leqslant z$ and $y \in J$. Then $y \cdot z=0 \in J$ (and $y * z=0 \in J$ ). Thus $z \in J$ by (3) (by (1) respectively).

If we use the labels

$$
\cdot(z, J)=\{y \in A: y \cdot z \in J\} \text { and } *(z, J)=\{y \in A: y * z \in J\},
$$

introduced and used in [8] and [9], we can conclude that as a consequence of the preceding theorem, the following is valid

Corollary 3.2. $J$ be a pseudo-UP ideal in a pseudo-UP algebra $\mathfrak{A}$. Then

(6) $(\forall z \in A)(z \in J \Longrightarrow(\cdot(z, J) \subseteq J \wedge *(z, J) \subseteq J))$.

Based on the orientation expressed in article [18], we introduce the concept of pseudo-UP filters as follows

Definition 3.2. A nonempty subset $F$ of a pseudo-UP algebra $\mathfrak{A}$ is called a pseudo-UP filter of $\mathfrak{A}$, if it satisfies the following properties:

(pF1) $0 \in F$;

(pF2) $(\forall x, y \in A)((x \in F \wedge x \cdot y \in F \Longrightarrow y \in F)$; and

(pF3) $(\forall x, y \in A)((x \in F \wedge x * y \in F \Longrightarrow y \in F)$.

From this determination, immediately follows:

Proposition 3.1. Let $F$ be a pseudo-UP filter in a pseudo-UP algebra $\mathfrak{A}$. Then

$(\forall x, y \in A)((x \in F \wedge x \leqslant y) \Longrightarrow y \in F)$.

Proposition 3.2. A nonempty subset $F$ of a pseudo-UP algebra $A$ is a pseudo-UP filter in $\mathfrak{A}$ if and only if $0 \in F$ and holds

(F4) $(\forall z \in A)(z \in F \Longrightarrow(\cdot(z, F) \subseteq F \wedge *(z, F) \subseteq F))$. 
Theorem 3.2. If $F$ is a pseudo-UP filter of a pseudo-UP algebra $\mathfrak{A}$, then

(7) $(\forall x, y, z \in A)((x \in F \wedge y \in F \wedge z * y \leqslant x) \Longrightarrow z \in F)$ and,

(8) $(\forall x, y, z \in A)((x \in F \wedge y \in F \wedge z \cdot y \leqslant x) \Longrightarrow z \in F)$.

Proof. Suppose that $F$ is a pseudo-UP filter of $\mathfrak{A}$ and let $x, y, z \in A$ be arbitrary elements.

Suppose that $z * y \leqslant x$. Then $(z * y) \cdot x=0 \in F$. Thus $z * y \in \cdot(x, F) \subseteq F$. It follows that $z \in *(y, F) \subseteq F$. Therefore, the condition (8) is proved.

Now let $x \in F, y \in F$ and $z \cdot y \leqslant x$ be hold. Then $(z \cdot y) * x=0 \in F$, and thus $z \cdot y \in *(x, F) \subseteq F$. Hence $z \in \cdot(y, F) \subseteq F$, which shows the condition (8).

\section{Final observation}

In the study of algebraic substructures of UP-algebras, this author took part with his texts ([12-16]). And this text should be seen as a continuation of these his efforts.

Looking at the [8-10], some types of pseudo-UP filters one can be introduced in pseudo-UP algebras. For example: one type of pseudo-UP filters can be introduced by requiring that the set $F$ satisfies $0 \in F$ and the following conditions

$(\forall x, y, z \in A)((((x \cdot y) * y) \cdot z \in F \wedge z \in F) \Longrightarrow x * y \in F)$ and

$(\forall x, y, z \in A)((((x * y) \cdot y) * z \in F \wedge z \in F) \Longrightarrow x \cdot y \in F)$.

Another type of pseudo-UP filters could be a subset of $F$ of a pseudo-UP algebra $A$ if $0 \in F$ and the following holds

$(\forall y \in A)(y \in F \Longrightarrow \cdot(y, F) \cap *(y, F) \subseteq F)$.

Further, drawing on the ideas in article [10], some other similar conditions could be analyzed.

\section{References}

[1] Dudek, W. A., Jun, Y. B.: Pseudo-BCI algebras. East Asian Math. J., 24(2), 187--190 (2008).

[2] Dymek, G.: Atoms and ideals of pseudo-BCI-algebras. Comment. Math., 52(1), 73--90 (2012).

[3] Georgescu, G., Iorgulescu, A.: Pseudo-BCK algebras: An extension of BCK-algebras. Combinatorics, computability and logic (Constanta, 2001), (pp. 97--114), Springer Ser. Discrete Math. Theor. Comput. Sci. Springer, London, 2001.

[4] Emanovský, P., Kühr, J.: Some properties of pseudo-BCK-and pseudo-BCI-algebras. Fuzzy Sets and Systems, 339, 1-16 (2018).

[5] Iampan, A.: A new branch of the logical algebra: UP-algebras. J. Algebra Relat Top., 5(1), 35--54 (2017).

[6] Iorgulescu, A.: On pseudo-BCK algebras and porims. Sci. Math. Japonicae Online, 10, 293--305 (2004).

[7] Iorgulescu, A.: Classes of pseudo-BCK algebras - Part I. J. of Mult.-Valued Logic Soft Comput., 12, 71--130 (2006).

[8] Jun, Y. B., Kondo, M., Kim, K. H.: Pseudo-ideals of pseudo-BCK algebras. Sci. Math. Japonicae Online, 8, 87-91 (2003).

[9] Jun, Y. B., Kim, H. S., Neggers, J.: On pseudo-BCI ideals of pseudo-BCI algebras. Mat. Vesnik, 58(1), 39--46 (2006).

[10] Jun, Y. B., Iampan, A.: Shift UP-filters and decompositions of UP-filters in UP-Algebras. Missouri J. Math. Sci., 31(1), 36-45 (2019).

[11] Lee, K. J., Park, C. H.: Some ideals of pseudo-BCI-algebras. J. Appl. Math. Inform., 27(1-2), 217--231 (2009).

[12] Romano, D. A.: Proper UP-filters in UP-algebra. Universal J. Math. Appl., 1(2), 98-100 (2018).

[13] Romano, D. A.: Notes on UP-ideals in UP-algebras. Comm. Advan. Math. Sci., 1(1), 35-38 (2018).

[14] Romano, D. A.: Some properties of proper UP-filters of UP-algebras. Fund. J. Math. Appl., 1(2), 109-111 (2018).

[15] Romano, D. A.: Some decompositions of UP-ideals and proper UP-filters. Math. Advan. Pure Appl. Sci., 2(1), 16-18 (2019). 
[16] Romano, D. A.: Pseudo-valuations on UP-algebras. Universal J. Math. Appl., 2(3), 138-140 (2019).

[17] Romano, D. A.: Pseudo-UP algebras, An introduction. Bull. Int. Math. Virtual Inst., 10(2), 349-355 (2020).

[18] Somjanta, J., Thuekaew, N. Kumpeangkeaw, P., Iampan, A.: Fuzzy sets in UP-algebras. Ann. Fuzzy Math. Inform., 12(6), 739-756 (2016).

[19] Walendziak, A.: On axiom systems of pseudo-BCK algebras. Bull. Malays. Math. Sci. Soc. (2), 34(2), 287--293 (2011).

\section{Affiliations}

DANIEL A. ROMANO

ADDRESS: International Mathematical Virtual Institute 6, Kordunaşka Street, 78000 Banja Luka, Bosnia and Herzegovina.

E-MAIL: bato49@hotmail.com

ORCID ID: 0000-0003-1148-3258 